\title{
NON-SURGICAL TREATMENT OF PEYRONIE'S DISEASE: A COMPREHENSIVE REVIEW
}

DOI: 10.36740/WLek202103129

\author{
Volodymyr A. Chernylovskyi' ${ }^{1}$ Denis V. Krakhotkin ${ }^{2}$, Viktor P. Chaikovskyi ${ }^{1}$ \\ ${ }^{1}$ CITY CLINICAL HOSPITAL № 4, DNIPRO, UKRAINE \\ ${ }^{2}$ OUTPATIENT CLINIC, CENTRAL DISTRICT HOSPITAL, KAMENOLOMNI, RUSSIA
}

\begin{abstract}
This review article focuses on conservative treatment options, topical, intralesional therapy, traction and vacuum therapy. A PubMed database search was performed for studies that were published between 1948 and 2019. Search keywords included "Peyronie's disease," "conservative therapy," "traction treatment," "extracorporeal shock wave therapy," "topical and oral therapies," and "vaccum therapy." Clinical trials in men with Peyronie's disease and scientific articles relating to pharmacologic data were included in the review. When possible, large, randomized, and well-designed trials were selected. Non-English-language articles were excluded.
\end{abstract}

KEY WORDS: Peyronie's disease, Intralesional treatment, penile curvature, Oral therapy, Penile traction, extracorporeal shock wave therapy

Wiad Lek. 2021;74(3 p.l):539-545

\section{INTRODUCTION}

Peyronie's disease (PD) is a fibrotic condition of the tunica albuginea (TA) characterized by excessive accumulation collagen fibers and other components extracellular matrix (ECM), avoiding of apoptosis myofibroblasts during the abnormal wound healing process, cytokine dysregulation and local inflammatory changes with formation inelastic penile plaques [1,2]. François Gigot de la Peyronie first described this condition in 1743 , but the pathogenesis is remaining unclear and enigmatic [3]. This disease has an acute (active) and chronic stable phases with different clinical course. In the acute phase, with duration, up to 18 months after the onset of PD occurs the inflammatory changes in TA due to repeated microtrauma during sexual intercourse. Insufficient activation of fibrinolysis and the fibroblast activation leads to a rapid growth plaque. In this period, the patient with PD notes the pain in flaccid state and painful erection, palpable nodes, and development of penile deformation. In the chronic phase, the pain generally disappears, and the formed plaques are stabilized, causing a penile curvature, penile deformities (such as hourglass deformity, hinge effect), and the shortening length of the penis $[4-5,8]$.

\section{THE AIM}

This review article focuses on conservative treatment options, topical, intralesional therapy, traction and vacuum therapy. A PubMed database search was performed for studies that were published between 1948 and 2019. Search keywords included "Peyronie's disease," "con- servative therapy," "traction treatment," "extracorporeal shock wave therapy," "topical and oral therapies," and "vaccum therapy." Clinical trials in men with Peyronie's disease and scientific articles relating to pharmacologic data were included in the review. When possible, large, randomized, and well-designed trials were selected. Non-English-language articles were excluded.

\section{MATERIALS AND METHODS}

The prevalence of PD ranges from 0.4 to $20.3 \%$, considering men with concomitant diabetes mellitus and erectile dysfunction. Peyronie's disease remains an under-diagnosed problem due to a paucity of epidemiological data of prevalence $[4,6,10]$. In the active inflammatory stage of PD should use oral, intralesional, and topical therapies despite controversial results and weak or moderate strength of recommendation in EAU and AUA guidelines. This is especially relevant in patients who have contraindications for surgical treatment due to severe concomitant somatic diseases.[7,9]. The oral medications available to treat Peyronie's disease are relatively inexpensive and are well-tolerated without adverse events. However, conservative treatment, when prescribed alone is not as effective as other modalities, such as intralesional injection and mechanical therapies. For any oral agent proposed for treatment of Peyronie's, the dose or tissue concentration that must reach in the tunica albuginea have a crucial role. The therapy with traction or vacuum devices may represent a viable therapeutic option for treating PD. 


\section{REVIEW AND DISCUSSION}

\section{ORAL MEDICATIONS}

\section{VITAMIN E (TOCOPHEROL)}

Vitamin $\mathrm{E}$ is a fat-soluble compound with potent antioxidant action, which was first suggested for the treatment of Peyronie's disease in 1948 by Scott and Scardino[11]. It is assumed that vitamin $\mathrm{E}$ decreases the collagen deposition by reducing free oxygen radicals, inhibition of TGF- $\beta 1$, and have an anti-inflammatory and anti-cyclooxygenase- 2 (COX-2) property $[12,13]$. Most urologists commonly prescribe it in dose $600 \mathrm{mg}$ per day because of its low cost, safety, and full availability. There are some clinical trials of vitamin $\mathrm{E}$ with conflicting results concerning the reduction of penile pain and deformity. In one study, Safarinejad et al. did not show significant improvement in pain, curvature, or plaque size in patients with PD treated with vitamin $\mathrm{E}$ compared with placebo[14]. Paulis et al. showed that vitamin E in combination therapy could lead to a significant improvement of penile curvature and reduction plaque size[15].

\section{TAMOXIFEN}

Tamoxifen is a nonsteroidal estrogen receptor antagonist that can block TGF- $\beta 1$-signaling through Smad- dependent and Smad-independent manner[16,17]. Its anti-fibrotic activity has confirmed in several studies. El-Shazly et al. showed that tamoxifen effectively prevents the recurrence of urethral stricture after internal urethrotomy and improvement of Qmax and IPSS score compared with the control group [18].In the rat model of Peyronie's disease, Ilg $\mathrm{MM}$, et al demonstrated that the combination of vardenafil and tamoxifen inhibited the myofibroblast transformation and ECM production[19].

Given this fact, Ralph et al. first demonstrated that tamoxifen is effective for the treatment of Peyronie's disease in 1992, and by now, there are few clinical trials about of effectiveness of tamoxifen as a part of combination therapy. In one study, Park et al. showed that the tamoxifen, together with acetyl-L-carnitine and phosphodiesterase type 5 inhibitor, significantly improved penile curvature $[20,21]$. The monotherapy tamoxifen will not lead to the significant improvement of penile deformity. This fact has demonstrated in a small placebo-controlled randomized study in 25 patients, which compared tamoxifen with placebo; no significant changes in penile curvature noted in either group [22]. The long-term use of tamoxifen can lead to such adverse events as constipation, hot flashes, atrial dysrhythmia, hypotension, superficial phlebitis, bone pain, decreased libido[23].

\section{POTASSIUM PARA-AMINOBENZOATE (POTABA)}

Potassium para-aminobenzoate is the agent with the antifibrotic activity, which first used for treatment PD by Zarafonetis and Horrax in 1953 [24]. It is believed that this substance exerts their antifibrotic properties through the improvement of tissue oxygenation, an increase of the secretion of glycosaminoglycans, and enhancement of the activity of monoamine oxidases with further decreasing local levels of serotonin[25]. In a prospective, randomized, double-blind, placebo-controlled, multicentre study in 103 patients with Peyronie's disease, Weidner et al. showed that Potaba could lead to a significant reduction in plaque size, but not in penile curvature or pain [26]. However, the treatment with Potaba can cause gastrointestinal adverse effects and skin photosensitization. Roy et al. reported a case report of acute hepatitis associated with the treatment with Potaba, and after 4 months of discontinuation of this agent, the patient noted a full biochemical recovery [27].

\section{PHOSPHODIESTERASE-5 INHIBITORS (PDE-5I)}

As known, nitric oxide (NO) and cyclic GMP have an anti-fibrotic activity by preventing excessive ECM deposition and myofibroblast transformation through inhibition TGF- $\beta 1$-signaling. The anti-fibrotic effect of PDE5 inhibitors on PD plaque is associated with increasing the levels of circulating NO caused by inhibition of cGMP degradation to GMP $[28,29]$. This fact has confirmed in several studies of penile fibrosis in the animal model. Ferrini et al. demonstrated that the long-term use of a high dose of the vardenafil more effectively reduced the collagen/smooth muscle and collagen III/I ratios and the numbers of myofibroblasts in experimental PD-like plaque in the rat [30]. In another study in the diabetic rat, $\mathrm{Li}$ et al. showed that concomitant administration of P144-antagonist peptide of TGF- $\beta 1$ and sildenafil improved the cavernosal fibrosis[31]. Gonzalez-Cadavid et al. have repeatedly proposed that the continuous long-term administration of PDE5 inhibitors may improve Peyronie's disease[28,32]. In one study, Ozturk et al. showed that ongoing administration of sildenafil $50 \mathrm{mg}$ significantly reduced the size of the plaque, and penile pain was in $33.3 \%$ and $66.6 \%$ cases, respectively[33]. In another study, Palmieri et al. reported that extracorporeal shock wave therapy neither alone nor in combination with tadalafil $5 \mathrm{mg}$ once daily was able to significantly improve plaque size and curvature degree in patients with Peyronie's disease[34]. Recently, Cocci et al. demonstrated that the combination of sildenafil 25 $\mathrm{mg}$ and collagenase of Clostridium hystoliticum $(\mathrm{CCH})$ significantly improved the penile curvature compared with $\mathrm{CCH}$ alone[35].

\section{COLCHICINE}

Colchicine is most commonly used for treating acute attacks of gout with anti-microtubule and anti-inflammatory properties. It is proposed that colchicine treat Peyronie's disease through inhibition of microtubule polymerization with a subsequent reduction in collagen synthesis, down-regulating of TGF- $\beta$ expression, and diminishing of inflammatory mediators $[12,36]$. Prieto Castro et al. reported that combined use of vitamin $\mathrm{E}$ and colchicine improved 
the penile curvature and plaque size in the early stage of Peyronie's disease[37].In one study, Akman demonstrated that lateral curvature is the most commonly changed deformity that mostly shifts to the dorsal side of the penis after treatment with colchicine[38]. In another prospective, randomized, single-blind study, Toscano et al. showed the absence of any significant differences between treatment with thiocolchicine and verapamil in an improvement of the penile curvature and reducing plaque size [39].

\section{L-CARNITINE}

$\mathrm{L}$-carnitine is a derivate of 6-N-trimethyl-lysine with antioxidant, anti-inflammatory, and anti-proliferative activity. It is assumed that carnitine reduces intracellular calcium levels in endothelial cells and suppresses fibroblast proliferation and ECM production via stimulation of NO-synthase $[40,41]$. In one study, Safarinejad et al showed that propionyl-L-carnitine alone or in combination with vitamin $\mathrm{E}$ had no significant improvement in penile curvature, plaque size, and pain in early stages of Peyronie's disease[42]. Recently, Park et al in another study showed that combined therapy with tamoxifen $(20 \mathrm{mg})$, acetyl-L-carnitine (300 $\mathrm{mg}$ ) twice daily, and phosphodiesterase type 5 inhibitor had a better response in men with penile curvature less than $30^{\circ}$ [43]. Ciociola et al. reported case report of a 20 -years-old Caucasian male where along with tadalafil, pentoxifylline, and $\mathrm{L}$-arginine the propionyl-L-carnitine decreased PD plaque size compared with intracavernosal verapamil alone [44].

\section{PENTOXIFYLLINE}

Pentoxifylline (PTX) is a nonspecific cAMP-PDE inhibitor with anti-inflammatory and anti-fibrotic properties. Several studies demonstrated that the pentoxifylline can inhibit fibroblast proliferation, TGF- $\beta 1$ expression, ECM deposition, and increases fibrinolytic activity $[45,46]$. In the culture of tunica albuginea-derived fibroblasts, Lin et al showed that pentoxifylline decreased the expression of Smad1/5 proteins and enhanced phosphorylation of the inhibitory Smad 6 protein [47]. Smith et al found that pentoxifylline diminished or stabilized the calcium content in PD plaque in $91.9 \%$ cases [48]. In one study, Alizadeh et al. demonstrated that combined therapy significantly improved penile curvature, plaque size, and the recovery rate of erectile dysfunction compared with pentoxifylline or verapamil alone[49].

\section{TOPICAL TREATMENT}

\section{TOPICAL VERAPAMIL}

Verapamil is one of the calcium channel blockers, which is widely used in cardiology for the treatment of hypertension, atrial fibrillation, and other cardiac rhythm disturbances [50]. Several studies showed that verapamil can inhibit the synthesis and secretion of the extracellular matrix and induces synthesis of procollagenase of fibroblasts. The verapamil also inhibits pro-inflammatory cytokines, TGF- $\beta 1$, and cellular proliferation of fibroblasts with further depolymerization of actin filaments. Eventually, it leads to alteration of cell shape, apoptosis, and reduction of ECM production in fibrous tissue[51,52]. Despite these useful properties of verapamil, there is no evidence that topical administration can reach high levels in tunica albuginea with enough concentration for clinically meaningful effect[53]. However, in one placebo-controlled pilot study, Fitch et al. showed that topical verapamil significantly improved the penile curvature, decreased the PD plaque size, reduced pain syndrome and increased the quality of erection in $61.1 \%, 84.7 \%, 100 \%$ and $81.8 \%$ cases, respectively at nine-month of treatment[54].

\section{EXTRACORPOREAL SHOCK WAVE THERAPY}

Extracorporeal shock wave therapy (ESWT) is a non-invasive method that uses acoustic waves in a pulsatile manner to treat Peyronie's disease. The exact mechanism of action is still not well understood. It is proposed that ESWT works by remodeling the penile plaque, resulting in a local enhancement of macrophage activity through stimulation of inflammatory reaction with subsequent lysis of fibrotic tissue and increasing of angiogenesis around PD plaque. Eventually, these effects may lead to reducing plaque size, diminishing penile deformity, and alleviating pain [55-57].In one study, Chitale et al. demonstrated that there were no significant differences between ESWT and sham therapy in an improvement of penile curvature and pain [58].In another placebo-controlled, randomized study, Hatzichristodoulou et al. showed that in $40 \%$ cases, ESWT had led to worsening of penile deviation, and there were not any differences in plaque size between the intervention group and placebo $(p=0.33)$ [59]. In one meta-analysis, Gao et al. demonstrated that ESWT reduces penile plaque size and relieves pain syndrome but not improve penile curvature and sexual function[60 ].However, Shimpi et al. reported that ESWT improved pain and penile curvature and reduced PD plaque[61]. In another recent study, Di Mauro et al. demonstrated that ESWT decreased plaque size, penile curvature, and pain assessed by visual analogue scale $(\mathrm{p}<0.001)$. They also showed that ESWT might improve an erectile function measured by IIEF and increase a penile length in erection $(\mathrm{p}<0.001)$ [62].

\section{MECHANICAL THERAPY}

\section{TRACTION DEVICES}

The mechanotransduction or gradual extension of tissue by traction in patients with PD increases the activity of collagenases and metalloproteinases. Ultimately, it leads to the formation of new connective tissue by cellular proliferation and softening of PD plaque. One of the chief complaints in patients with PD is the loss of penile length; thus, penile traction therapy is a reasonable treatment option $[63,64]$.In 
Table 1. Intralesional therapies for treatment of Peyronie's disease

\begin{tabular}{ccc}
\hline Intralesional therapy & Mechanisms of action & Side effects \\
\hline Interferon alpha $2 \mathrm{~b}$ & $\begin{array}{c}\text { Antifibrotic activity, } \\
\text { Immune system stimulation }\end{array}$ & $\begin{array}{c}\text { Flu-like symptoms, sinusitis, } \\
\text { Penile swelling }\end{array}$ \\
\hline Hyaluronic acid & $\begin{array}{c}\text { Anti-inflammatory properties } \\
\text { Antioxidant activity }\end{array}$ & Small ecchymoses \\
\hline Collagenase, Clostridium, Histolyticum & Degradation of collagen & Penile bruishing, Penile swelling \\
\hline
\end{tabular}

one study, Ziegelmann et al. demonstrated that penile traction therapy with the RestoreX device for 30 to 90 minutes per day significantly improved the penile curvature and length in men with PD without serious adverse effects[65]. Moncada et al. demonstrated that the use of the Penimaster PRO significantly reduced the penile curvature in ranging from 200 to 500 , with $51.4 \%$ improvement compared with a non-intervention group[66].In a nonrandomized prospective controlled trial, Martínez-Salamanca et al. showed that penile traction therapy decreased the penile curvature from $33^{\circ}$ at baseline to $15^{\circ}$ at six months and $13^{\circ}$ at nine months of treatment in patients with PD in the acute phase [67](Table 1). Recently, Ibrahim et al. reported that penile traction therapy combined with pentoxifylline or colchicine significantly decreased penile curvature and PD plaque size[68].

\section{VACUUM DEVICES}

Vacuum therapy is a non-invasive method of treatment Peyronie's disease with a similar mechanism of action as for penile traction therapy. It based on the reordering of disordered collagen in the inelastic scar through mechanotransduction with the further active remodeling of tissue $[69,70]$.In a rat model, $\mathrm{Li}$ et al. showed that the vacuum erection device can reduce Peyronie's-like plaque size and decreased the expression of TGF- $\beta 1$ and SMAD $2 / 3$ proteins [71].In one study, Raheem et al. demonstrated that the vacuum pump therapy reduced penile curvature by 5-25 degrees and may reduce the number of patients going on to surgery [72]. In another randomized,open-label study, Ralph et al. showed that vacuum-pump therapy both alone or in combination with collagenase Clostridium histolyticum $(\mathrm{CCH})$ treatment might improve the penile curvature[73].

\section{INTRALESIONAL TREATMENT}

\section{INTRALESIONAL INTERFERON A2B}

Interferon $a-2 b$ (IFN- $a-2 b)$ have been shown to decrease fibroblast proliferation and produce collagen and other ECM proteins, through increasing the levels of collagenase and inhibiting of metalloproteinases, which inhibit collagenase[74]. Under these properties of IFN- $a-2 b$, it is widely applied in the treatment of hypertrophic scars, liver fibrosis, and other fibrotic conditions related to fibroblast dysregulation[75,76].In one study, Stewart et al. showed that intralesional interferon- $\alpha 2 \mathrm{~b}$ provided greater than $20 \%$ reduction in penile curvature with a total response rate of $91 \%$ independently from the localization of PD-plaque[77]. Trost et al. reported similar findings after intralesional interferon- $\alpha 2 \mathrm{~b}$ injections in patients with penile curvature less than 30-degree and without impacting penile vascular parameters[78]. In a prospective study, Sokhal et al. reported a significant improvement of plaque volume and penile curvature after treatment with intralesional IFN- $\alpha 2 b[79]$.

\section{HYALURONIC ACID (HA)}

Hyaluronic acid (HA) plays an essential role in the regeneration of the cellular components during the wound healing process. Hyaluronan (HA) is also active in the ECM reorganization, cytokine, and growth factor adhesion as well as recruitment of matrix metalloproteinases (MMPs). Hyaluronic acid (HA) exists in two forms: high molecular weight hyaluronan with anti-inflammatory properties and HA fragments with low molecular weight, which promote inflammation. The matrix enriched with high molecular weight hyaluronan acts as an alternate protective target for reactive oxygen and nitrogen species (ROS/RNS) generated during inflammation and limits penetration of those species to the cell membrane[80].

Based on these data, it was proposed the intralesional administration of $\mathrm{HA}$ with promising effects in improvement of Peyronie's disease.In one prospective,double-blinded, randomized study, Favilla et demonstrated that treatment with intralesional hyaluronic acid (HA) had greater efficacy in terms of penile curvature compared with intralesional verapamil. Gennaro et al. reported that intralesional HA significantly reduced the plaque volume and penile curvature compared with the control group.In a prospective, interventional, multicenter pilot study, Zucchi et al. showed that intralesional administration of hyaluronic acid (HA) significantly improved plaque size, penile curvature, and overall sexual satisfaction.

\section{INTRALESIONAL COLLAGENASE CLOSTRIDIUM HISTOLYTICUM (CCH)}

The proteolytic properties of collagenase Clostridium histolyticum $(\mathrm{CCH})$ isolated from bacteria C.histolyticum were first described in the early 1950s. Gelbard is considered the first researcher who used the collagenase Clostridium histolyticum for the treatment of Peyronie's 
disease in the 1980s. In IMPRESS (Investigation for Maximal Peyronie's Reduction Efficacy and Safety Studies) I and II clinical trials, Gelbard et al. demonstrated that men treated with collagenase Clostridium histolyticum $(\mathrm{CCH})$ had a mean $34 \%$ improvement in penile curvature compared with a mean $18.2 \%$ improvement in the placebo group. These findings in further allowed to establish the current FDA-approved intralesional collagenase plus modeling protocol for stable PD.In one study, Yang et al. showed that treatment with collagenase Clostridium histolyticum $(\mathrm{CCH})$ reduced penile curvature on 15.4 degrees compared with baseline but without significant changes in penile pain. Nguyen et al. conducted a retrospective analysis of patients in acute phase Peyronie's disease treated with $\mathrm{CCH}$ and found that there was no significant difference in final change in curvature $16.7^{\circ}$ vs. $15.6^{\circ}$ between the acute and stable phases of PD, respectively. The results of IMPRESS I and II trials are replicated in some other studies. In the multi-institutional analysis, Hellstrom et al. showed that the 502 patients who completed 4 or more cycles curvature improved from a mean of 49.7 degrees before to 32.7 degrees after treatment, a $33 \%$ improvement from baseline $(\mathrm{p}<0.0001)$ with a low rate of complications. The treatment is effective in atypical cases of Peyronie's disease. In prospective study, Alom et al. demonstrated that after treatment with collagenase Clostridium histolyticum $(\mathrm{CCH})$, ventral and lateral curvatures experienced greater relative improvements in curvature compared to dorsal (ventral 29.5 degrees [49\%], lateral 11.4 [38\%], dorsal 15.0 [25\%], respectively). In one retrospective analysis of 65 patients with $\mathrm{PD}$, Cocci et al. demonstrated that $\mathrm{CCH}$ improved penile curvature (PC) on $15^{\circ}-20^{\circ}$ degree compared with baseline regardless of the form of penile deformity (ventral, hourglass and shortening) without significant differences between groups. Fernández-Pascual et al. showed that combination of percutaneous needle tunneling (PNT) and CCH significantly improved the penile curvature compared with $\mathrm{CCH}$ alone $36.2 \pm$ $12.5 \%$ vs. $28.1 \pm 14.5 \%$, respectively ( $\mathrm{p}=0.001)$. However, the rate of success of $\mathrm{CCH}$ therapy may be associated with a degree of severity of plaque calcification. Wymer et al. showed that the patients with a noncalcified plaque and penile curvature $\geq 60^{\circ}$ had a good response on treatment with $\mathrm{CCH}$ compared with moderate or severe $(>1 \mathrm{~cm})$ calcination.Despite the enormous clinical effect of collagenase Clostridium Histolyticum (CCH) in improving penile curvature, it is impossible not to take into account possible serious complications that may require surgical intervention. In one study, Beilan et al. reported that of the 102 patients with Peyronie's disease, $12(11,8 \%)$ had a hematoma and $5(4,9 \%)$ penile fracture after treatment with $\mathrm{CCH}$. Using the modified injection procedure, termed the «fan» technique, Amighi et al. demonstrated the absence of corporeal ruptures and minimal percentage of hematomas, but bruising or swelling was seen in 54.6\% and $27.0 \%$ of cases, respectively. Intralesional therapies are summarized in table 1.

\section{CONCLUSIONS}

PD remains a challenge for urologists. Evidence suggests that, for oral therapy, a combination of drugs produces better results but current evidences from AUA and EAU guidelines are weak. Although on the improvement of pain syndrome, extracorporeal shock wave therapy is still unproven and necessary more RCT. Clostridium histolyticum $(\mathrm{CCH})$ is the only FDA-approved treatment option available. Penile traction and vacuum therapy may improve penile curvature.

\section{REFERENCES}

1. Garaffa G., Trost L.W., Serefoglu E.C. et al. Understanding the course of Peyronie's disease. Int J Clin Pract. 2013 ;67(8):781-8. doi: 10.1111/ ijcp.12129.

2. Aliperti L.A. , Mehta A. Peyronie's Disease: Intralesional Therapy and Surgical Intervention Curr Urol Rep. 2016 ;17(9):60. doi:10.1007/ s11934-016-0622-2.

3. De Young L.X., Bella A.J., O'Gorman D.B. et al Protein biomarker analysis of primary Peyronie's disease cells. J Sex Med 2010;7:99-106. D0I: 10.1111/j.1743-6109.2009.01556.x

4. Langston J.P., Carson I.I.I.C.C. Peyronie's disease: Review and recent advances. Maturitas 2014. http://dx.doi.org/10.1016/j.ma...

5. Levine L.A., Larsen S.M. Surgical correction of persistent Peyronie's disease following collagenase clostridium histolyticum treatment. J Sex Med. 2015 ;12(1):259-64. doi: 10.1111/jsm. 12721.

6. Chung E., Ralph D., Kagioglu A. et al. Evidence-Based Management Guidelines on Peyronie's Disease. J Sex Med. 2016;13:905-923. doi: 10.1016/j.jsxm.2016.04.062.

7. Carson C.C., Levine L.A. Outcomes of surgical treatment of Peyronie's disease. BJU Int. 2014;113(5):704-13. doi: 10.1111/bju.12565.

8. Levine L.A., Larsen S.M. Surgery for Peyronie's disease. Asian J Androl. 2013;15(1):27-34. doi:10.1038/aja. 2012.92.

9. Twidwell J., Levine L. Topical treatment for acute phase Peyronie's disease utilizing a new gel, $\mathrm{H}-100$ : a randomized, prospective, placebocontrolled pilot study. Int J Impot Res. 2016 ;28(2):41-5. doi:10.1038 / ijir.2015.22.

10. Nehra A., Alterowitz R., Culkin D.J. etal. Peyronie's Disease: AUA Guideline. The Journal of Urology ${ }^{\circledast} .2015$. doi: 10.1016/j.juro.2015.05.098.

11. Scott W.W., Scardino P.L. A new concept in the treatment of Peyronie's disease. South Med J. 1948;41:173-7.

12. Paulis $G$., Brancato $T$. Inflammatory mechanisms and oxidative stress in Peyronie's disease: therapeutic «rationale» and related emerging treatment strategies.Inflamm Allergy Drug Targets. 2012;11(1):48-57.

13. Tasanarong A., Kongkham S., Duangchana S. et al Vitamin E ameliorates renal fibrosis by inhibition of TGF-beta/Smad2/3 signaling pathway in UU0 mice.J Med Assoc Thai. 2011;94(7):S1-9.

14. Safarinejad M.R., Hosseini S.Y., Kolahi A.A. Comparison of Vitamin E and Propionyl-L-Carnitine, Separately or in Combination, in Patients With Early Chronic Peyronie's Disease: A Double-Blind, Placebo Controlled, Randomized Study. Journal of Urology. 2007;178(4):1398-1403. doi: 10.1016/j.juro.2007.05.162.

15. Paulis G., Brancato T., D'Ascenzo R. et al. Efficacy of vitamin E in the conservative treatment of Peyronie's disease: legend or reality? A controlled study of 70 cases. Andrology. 2013;1(1):120-128.

16. Yan P., Tang H., Chen X. et al.Tamoxifen attenuates dialysate-induced peritoneal fibrosis by inhibiting GSK-3 $\beta / \beta$-catenin axis activation.Biosci Rep. 2018;38(6). doi: 10.1042/BSR20180240. 
17. KimD., LeeA.S., JungY.J. et al.Tamoxifen ameliorates renal tubulointerstitial fibrosis by modulation of estrogen receptor a-mediated transforming growth factor- $\beta 1 /$ Smad signaling pathway.Nephrol Dial Transplant. 2014 ;29(11):2043-53. doi: 10.1093/ndt/gfu240.

18. El-Shazly M., Hodhod A., Selim M. et al.The Effectiveness of Tamoxifen in the Prevention of Recurrent Urethral Strictures Following Internal Urethrotomy.Urol Int. 2018;101(4):472-477. doi: 10.1159/000493173.

19. Ilg M.M. et al. Antifibrotic Synergy Between Phosphodiesterase Type 5 Inhibitors and Selective Oestrogen Receptor Modulators in Peyronie's Disease Models. Eur Urol. 2018. https://doi.org/10.1016/j.euru...

20. Ralph D.J., Brooks M.D., Bottazzo G.F. et al. The treatment of Peyronie's disease with tamoxifen. Br J Urol. 1992;70:648-651.

21. ParkT.Y.,Jeong H.G.,ParkJ.J.etal.TheEfficacy of Medical Treatment of Peyronie's Disease: Potassium Para-Aminobenzoate Monotherapy vs. Combination Therapy with Tamoxifen, L-Carnitine, and Phosphodiesterase Type 5 Inhibitor. World J Mens Health. 2016;34(1):40-6. doi: 10.5534/wjmh.2016.34.1.40.

22. Teloken C., Rhoden E.L., Grazziotin T.M. et al. Tamoxifen versus placebo in the treatment of Peyronie's disease. J Urol. 1999;162:2003-5.

23. Wibowo E., Pollock P.A., Hollis N. et al. Tamoxifen in men: a review of adverse events.Andrology. 2016;4(5):776-88. doi: 10.1111/andr.12197.

24. Zarafonetis C.J., Horrax T.M. Treatment of Peyronie's disease with potassium para-aminobenzoate (potaba). J. Urol. 1959;81: 770-772.

25. HauckE.W., DiemerT., Schmelz H.U. et al. Critical analysis of nonsurgical treatment of Peyronie's disease. Eur Urol. 2006;49:987-97.

26. Weidner W., Hauck E.W., Schnitker J. Potassium para-aminobenzoate (POTABA) in the treatment of Peyronie's disease: a prospective, placebocontrolled, randomized study. Eur Urol 2005: 47(4):530-535.

27. Roy J., Carrier S. Acute hepatitis associated with treatment of Peyronie's disease with potassium para-aminobenzoate (Potaba).J Sex Med. 2008;5(12):2967-9. doi: 10.1111/j.1743-6109.2008.00918.x.

28. Gonzalez-Cadavid N.F., Rajfer J. Treatment of Peyronie's disease with PDE5 inhibitors: an antifibrotic strategy. Nat. Rev. Urol. 2010;7:215-221.

29. Iacono F., Prezioso D., Somma P. et al Histopathologically proven prevention of post-prostatectomy cavernosal fibrosis with sildenafil. Urol Int. 2008;80(3):249-52. doi: 10.1159/000127335.

30. Ferrini M.G., Kovanecz I., Nolazco E. et al. Effects of long-term vardenafil treatment on the development of fibrotic plaques in a rat model of Peyronie's disease. BJU Int. 2006;97: 625-633.

31. Li W.J., Wang H., Zhou J., Li B. et al. P144, A TGF- $\beta 1$ antagonist peptide, synergizes with sildenafil and enhances erectile response via amelioration of cavernosal fibrosis in diabetic rats.J Sex Med. 2013;10(12):2942-51. doi: 10.1111/jsm. 12325.

32. Gonzalez-Cadavid N.F., Rajfer J. The two phases of the clinical validation of preclinical translational mechanistic research on PDE5 inhibitors since Viagra's advent. A personal perspective.Int J Impot Res. 2019;31(2):5760. doi: 10.1038/s41443-018-0076-9.

33. Ozturk U., Yesil S., Goktug H.N. et al. Effects of sildenafil treatment on patients with Peyronie's disease and erectile dysfunction.Ir J Med Sci. 2014;183(3):449-53. doi: 10.1007/s11845-013-1036-5.

34. Palmieri A.,Imbimbo C., CretaM. et al Tadalafil once daily and extracorporeal shock wave therapy in the management of patients with Peyronie's disease and erectile dysfunction: results from a prospective randomized trial.Int J Androl. 2012;35(2):190-5. doi: 10.1111/j.1365-2605.2011.01226.x.

35. Cocci A., Cito G., Urzì D. et al. Sildenafil 25 mg ODT + collagenase Clostridium hystoliticum vs collagenase Clostridium hystoliticum alone for the management of Peyronie's disease: A matched-pair comparison analysis. J Sex Med. 2018;15:1472-1477. doi: 10.1016/j. jsxm.2018.08.012.
36. Tan R.B., Sangkum P., Mitchell G.C. et al Update on medical management of Peyronie's disease.Curr Urol Rep. 2014;15(6):415. doi: 10.1007/ s11934-014-0415-4.

37. Prieto Castro R.M., Leva Vallejo M.E., Regueiro Lopez J.C. et al. Combined treatment with vitamin $E$ and colchicine in the early stages of Peyronie's disease. BJU Int. 2003;91:522-4.

38. Akman T., Sanli 0., Uluocak N. et al The most commonly altered type of Peyronie's disease deformity under oral colchicine treatment is lateral curvature that mostly shifts to the dorsal side. Andrologia. 2011;43(1):28-33. doi: 10.1111/j.1439-0272.2009.01004.x.

39. Toscano L.Jr., Rezende M.V., Mello L.F. et al prospective, randomized, single - blind study comparing intraplaque injection of thiocolchicine and verapamil in Peyronie's Disease: a pilot study.Int Braz J Urol. 2016 ;42(5):1005-1009. doi: 10.1590/S1677-5538.IBJU.2015.0598.

40. Adeva-Andany M.M., Calvo-Castro I., Fernández-Fernández C. et al. Significance of I-carnitine for human health.IUBMB Life. 2017;69(8):578-594. doi: 10.1002/iub.1646.

41. CavalliniG.,BiagiottiG.Oral propionyl-L-carnitine and intraplaque verapamil in the therapy of advanced and resistantPeyronie's disease. BJUInt. 2002;89:895.

42. Safarinejad M.R., Hosseini S. Comparison of vitamin E and propionyl-Lcarnitine, separately or in combination, in patients with early chronic Peyronie's disease: a double-blind, placebo controlled, randomized study. J Urol. 2007;178:1398-403.

43. Park T.Y., Jeong H.G., Park J.J. et al.The Efficacy of Medical Treatment of Peyronie's Disease: Potassium Para-Aminobenzoate Monotherapy vs. Combination Therapy with Tamoxifen, L-Carnitine, and Phosphodiesterase Type 5 Inhibitor.World J Mens Health. 2016;34(1):406. doi: 10.5534/wjmh.2016.34.1.40.

44. Ciociola F., Colpi G.M. Peyronie's disease: a «triple oxygenant therapy». Arch Ital Urol Androl. 2013 ;85(1):36-40. doi: 10.4081/aiua.2013.1.36.

45. Safarinejad M.R., Asgari M. A double-blind placebo-controlled study of the efficacy and safety of pentoxyfylline in early chronic Peyronie's disease. BJU Int. 2010;106(2):240-8.

46. Shindel A.W., Lin G. Pentoxifylline attenuates transforming growth factor- beta-1-stimulated collagen deposition and elastogenesis in human tunica albuginea-derived fibroblasts part 1: impact on extracellular matrix. J Sex Med. 2010;7(6):2077-85.

47. Lin G., Shindel A.W., Banie L. et al. Pentoxifylline attenuates transforming growth factor-beta1-stimulated elastogenesis in human tunica albugineaderived fibroblasts part 2: Interference in a TGF-beta1/Smad-dependent mechanism and downregulation of AAT1. J Sex Med 2010;7:1787-97

48. Smith, J. F. et al. Pentoxifylline treatment and penile calcifications in men with Peyronie's disease. Asian J. Androl. 2011;13:322-325.

49. Alizadeh M., Karimi F., Fallah M.R. Evaluation of verapamil efficacy in Peyronie's disease comparing with pentoxifylline.Glob J Health Sci. 2014;6(7):23-30. doi: 10.5539/gjhs.v6n7p23.

50. Wang X., Lin Y., Chen L. et al.Treatment with verapamil for restoration of sinus rhythm in atrial fibrillation with rapid ventricular response: $A$ case report.Medicine (Baltimore). 2019;98(23):e15892. doi: 10.1097/ MD.0000000000015892.

51. Wang R., Mao Y., Zhang Z. et al.Role of verapamil in preventing and treating hypertrophic scars and keloids.Int Wound J. 2016;13(4):461-8. doi: 10.1111/iwj.12455.

52. Boggio R.F., Boggio L.F., Galvão B.L. et al Topical verapamil as a scar modulator. Aesthetic Plast Surg. 2014;38:968-75.

53. Martin D.J., Badwan K., Parker M. et al Transdermal application of verapamil gel to the penile shaft fails to infiltrate the tunica albuginea. J. Urol. 2002;168: 2483-2485. 
54. FitchW.P.3rd, EasterlingW.J., Talbert R.L. etal.Topical verapamil HCl, topical trifluoperazine, and topical magnesium sulfate for the treatment of Peyronie's disease — a placebo-controlled pilot study.J. Sex. Med. 2007;4:477-484.

55. Krieger J.R., Rizk P.J., Kohn T.P. et al. A Shockwave Therapy in Sex Med Rev. the Treatment of Peyronie's Disease. 2019;7(3):499-507. doi: 10.1016/j.sxmr.2019.02.001.

56. Hatzimouratidis K., Eardley I., Giuliano F. et al.EAU guidelines on penile curvature. Eur Urol. 2012;62(3):543-52. doi: 10.1016/j. eururo.2012.05.040.

57. Husain J., Lynn N.N., Jones D.K. et al. Extracorporeal shock wave therapy in the management of Peyronie's disease: initial experience. BJU Int. 2000;86:466-468.

58. Chitale S., Morsey M., Swift L. et al.Limited shock wave therapy vs sham treatment in men with Peyronie's disease: results of a prospective randomized controlled double-blind trial.BJU Int. 2010;106(9):1352-6. doi: 10.1111/j.1464-410X.2010.09331.x.

59. Hatzichristodoulou G. et al. Extracorporeal shock wave therapy in Peyronie's disease: results of a placebo-controlled, prospective, randomized, single-blind study. J. Sex. Med. 2013;10: 2815-2821.

60. Gao L., Qian S., Tang Z. et al. A meta-analysis of extracorporeal shock wave therapy for Peyronie's disease.Int J Impot Res. 2016;28(5):161-6. doi: $10.1038 / \mathrm{ijir} .2016 .24$.

61. Shimpi R.K., Jain R.J. Role of extracorporeal shock wave therapy in management of Peyronie's disease: A preliminary report.Urol Ann. 2016;8(4):409-417. doi: 10.4103/0974-7796.192100.

62. Di Mauro M., Russo G.I., Della Camera P.A. et al. Extracorporeal Shock Wave Therapy in Peyronie's Disease: Clinical Efficacy and Safety from a Single-Arm Observational Study.World J Mens Health. 2019;37(3):339346. doi: 10.5534/wjmh. 180100.

63. Chung E., De Young L., Solomon M., Brock G.B. Peyronie's disease and mechanotransduction: an in vitro analysis of the cellular changes to Peyronie's disease in a cell-culture strain system. J Sex Med. 2013; 10(5):1259-67. doi: 10.1111/jsm.12082.

64. Schaeffer A.J., Burnett A.L. Nonsurgical interventions for Peyronie disease: 2011 update. J Androl. 2012 ;33(1):3-14. doi: 10.2164/ jandrol.110.010561.

65. Ziegelmann M., Savage J., Toussi A., Alom M. et al. Outcomes of a Novel Penile Traction Device in Men with Peyronie's Disease: A Randomized, Single-Blind, Controlled Trial.J Urol. 2019. doi: 10.1097/ JU.0000000000000245.

66. Moncada I., Krishnappa P., Romero J. et al. Penile traction therapy with the new device'Penimaster PRO' is effective and safe in the stable phase of Peyronie's disease: a controlled multicentre study.BJU Int. 2019; 123(4):694-702. doi: 10.1111/bju.14602.

67. Martínez-Salamanca J.I., Egui A., Moncada I. et al. Acute phase Peyronie's disease management with traction device: a nonrandomized prospective controlled trial with ultrasound correlation. JSex Med. 2014 ;11(2):506-15. doi: 10.1111/jsm.12400.

68. Ibrahim A., Gazzard L., Alharbi M. et al. Evaluation of Oral Pentoxifylline, Colchicine, and Penile Traction for the Management of Peyronie's Disease.Sex Med. 2019. doi: 10.1016/j.esxm.2019.07.003.

69. Lin H., Liu C., Wang R. Effect of Penile Traction and Vacuum Erectile Device for Peyronie's Disease in an Animal Model.JS Sex Med. 2017;14(10):12701276. doi: 10.1016/j.jsxm.2017.08.011.

70. Chung E., De Young L., Solomon M. et al Peyronie's disease and mechanotransduction: an in vitro analysis of the cellular changes to Peyronie's disease in a cell-culture strain system.J Sex Med. 2013;10(5):1259-67. doi: 10.1111/jsm.12082.
71. Li J., Wang S., Qin F. et al.Reduction in Peyronie's-like plaque size using a vacuumerection device ina ratmodel of Peyronie's diseaseviatheTGF- $\beta / S M A D$ signalling pathway.Andrologia.2018;50(7):e13051.doi: 10.1111/and.13051.

72. Raheem A.A., Garaffa G., Raheem T.A. et al.The role of vacuum pump therapy to mechanically straighten the penis in Peyronie's disease. BJU Int. 2010;106(8):1178-80. doi:10.1111/j.1464-410X.2010.09365.x.

73. Ralph D.J., Abdel Raheem A., Liu G. Treatment of Peyronie's Disease With Collagenase Clostridium histolyticum and Vacuum Therapy: A Randomized, Open-Label Pilot Study. J Sex Med. 2017;14(11):14301437. doi: 10.1016/j.jsxm.2017.08.015.

74. Chong W., Tan R.B. Injectable therapy for Peyronie's disease.Transl Androl Urol. 2016;5(3):310-7. doi: 10.21037/tau.2016.03.15.

75. Berman B., Maderal A., Raphael B. Keloids and Hypertrophic Scars: Pathophysiology, Classification, and Treatment.Dermatol Surg. 2017;43(1):S3-S18. doi: 10.1097/DSS.0000000000000819.

76. Tamai H., Shingaki N., MoriY. et al. Low-Dose Pegylated Interferon a-2b Plus Ribavirin for Elderly and/or Cirrhotic Patients with Genotype 2 Hepatitis CVirus.Gut Liver. 2016;10(4):617-23. doi: 10.5009/gnl15193.

77. StewartC.A.,YafiF.A., Knoedler M.M. etal. Intralesional Injection of Interferona $2 \mathrm{~b}$ Improves Penile Curvature in Men with Peyronie's Disease Independent of Plaque Location.JUrol. 2015;194(6):1704-7. doi: 10.1016/j.juro.2015.06.096.

78. Trost L.W., Ates E., Powers M. et al.Outcomes of intralesional interferona2B for the treatment of Peyronie disease.J Urol. $2013 ; 190(6): 2194-9$. doi: 10.1016/j.juro.2013.05.022.

79. Sokhal A.K., Jain N.K.,Jhanwar A. etal. Prospectivestudy to evaluate the clinical outcome of intralesional interferon- $a 2 b$ in the management of Peyronie's disease.Urol Ann. 2018;10(2):154-158. doi: 10.4103/UA.UA_65_17.

80. Isık S., Taşkapıııoğlu M.Ö., Atalay F.0. et al Effects of cross-linked highmolecular-weight hyaluronic acid on epidural fibrosis: experimental study.J Neurosurg Spine. 2015;22(1):94-100. doi: 10.3171/2014.10.SPINE131147.

For the complete reference list, please, contact the corresponding author-Volodymyr A. Chernylovskyi-chernylovskyi@gmail.com.

\section{ORCID and contributionship:}

Volodymyr A. Chernylovskyi: 0000-0002-5795-1574 ${ }^{B, F}$

Denis V. Krakhotkin: 0000-0003-1540-6647 A,B,D,E,F

Viktor P. Chaikovskyi: 0000-0001-7974-3656 ${ }^{B, F}$

\section{Conflict of interest:}

The Authors declare no conflict of interest.

\section{CORRESPONDING AUTHOR Volodymyr A. Chernylovskyi \\ City Clinical Hospital No. 4 \\ 31 Blizhnya St., 49102 Dnipro, Ukraine \\ tel: +380973695672 \\ e-mail: chernylovskyi@gmail.com}

Received: 21.04 .2020

Accepted: 23.11 .2020

A - Work concept and design, B - Data collection and analysis, C - Responsibility for statistical analysis, D-Writing the article, $\mathbf{E}-$ Critical review, $\mathbf{F}-$ Final approval of the article 